\title{
Active Overt Obscured Gastrointestinal Bleeding Due to Ectasia of the Anterior Inferior Pancreaticoduodenal Artery and Its Endovascular Management in a Treated Case of Superior Mesenteric Artery Stenosis
}

\author{
Nischal G. Kundaragi ${ }^{1}$ Naresh Bhat ${ }^{2}$ \\ ${ }^{1}$ Department of Interventional Radiology, Aster CMI Hospital, \\ Bengaluru, Karnataka, India \\ 2Department of Gastroenterology and Liver Disease, Aster CMI \\ Hospital, Bengaluru, Karnataka, India \\ ${ }^{3}$ Department of Radiology, Aster CMI Hospital, Bengaluru, \\ Karnataka, India
}

J Clin Interv Radiol ISVIR 2018;2:207-210

The term overt obscure gastrointestinal bleeding (OGIB) is defined as patients presenting with either hematochezia or melena, in whom upper and lower endoscopic examinations in addition to a small bowel (SB) series (radiographic/ fluoroscopy examination of SB with positive contrast) did not reveal any source of bleeding in the gastrointestinal (GI) tract and may represent a source of bleeding outside the SB. ${ }^{1,2}$ In 85 to $90 \%$ of cases with OGIB, a source can be identified,; however, in 5 to $10 \%$, no source of bleeding is found. OGIB from the small intestines is difficult to localize. ${ }^{1}$ Small intestinal bleeding is among the less common causes of GI bleeds and accounts for $\sim 5$ to $10 \%$ of cases. According to the American College of Gastroenterology (ACG) clinical guidelines, in acute overt GI bleeding, conventional angiography should be performed on an emergency basis for hemodynamically unstable patients and computed tomography angiography (CTA) can be performed in hemodynamically stable patients to identify the site of bleeding and to guide further management. ${ }^{2}$ The present case report is a description of a rare case of acute overt massive upper GI bleed, 1 month after superior mesenteric artery stenting. Source of bleeding was not found on upper and lower GI endoscopy and CTA. Catheter-based angiography identified anterior inferior pancreaticoduodenal artery (AIPDA) ectasia, which was subsequently embolized successfully.

\section{Case Report}

A 57-year-old male patient presented with a history of postprandial abdominal pain for 9 months. On evaluation, he was found to have significant superior mesenteric artery (SMA) stenosis, with a Doppler velocity measuring up to

received

July 18,2018 accepted after revision

August 20, 2018

published online

November 27, 2018
$495 \mathrm{~cm} / \mathrm{s}$, suggestive of $>70 \%$ stenosis. This was confirmed on CTA. Angioplasty and stenting of the SMA were successfully performed under adequate periprocedural anticoagulation with intravenous administration of $5000 \mathrm{IU}$ of unfractionated heparin ( - Fig. 1). A balloon-expandable bare metal stent was placed in the SMA (7 mm diameter and $36 \mathrm{~mm}$ length, the Valeo stent; Bard Peripheral Vascular). Post procedure, peak systolic velocity on Doppler ultrasound had reduced to $<200 \mathrm{~cm} / \mathrm{s}$, and a significant reduction in abdominal pain was noted.

The patient was discharged on dual antiplatelet medications ( $75 \mathrm{mg}$ of clopidogrel and $150 \mathrm{mg}$ of aspirin once a day). One month later, the patient presented to the emergency department with severe melena. He was hemodynamically unstable on initial examination, blood pressure was $80 / 55 \mathrm{~mm} \mathrm{Hg}$, and hemoglobin was $4 \mathrm{~g} \%$. His platelet count was $216,000 / \mathrm{microL}$, serum bilirubin was $0.9 \mathrm{mg} / \mathrm{dL}$, serum amylase was $61.3 \mathrm{mg} / \mathrm{dL}$, prothrombin time (PT) was 14.3 seconds, and international normalized ratio (INR) was 1.28 . The antiplatelets were withheld. The patient was resuscitated with intravenous fluids and packed cells, and emergency upper and lower GI endoscopies were performed, which revealed scanty fresh blood in the duodenum and clots in the colon. No active bowel bleeding was observed. Enteroscopy was not performed in view of ongoing melena. A CTA did not reveal any significant vascular anomaly or source of active bleeding. In view of ongoing bleeding, a catheter angiogram was scheduled immediately with plans to embolize if required.

Catheter-guided mesenteric angiogram was performed using a 5F Cobra catheter (Cordis) and a coaxially introduced 2.7F microcatheter (Progreat 2.0; Terumo). Radiology
C2018 by Indian Society of Vascular and Interventional

License terms

$10.1055 / \mathrm{s}-0038-1676158$

ISSN 2457-0214. 

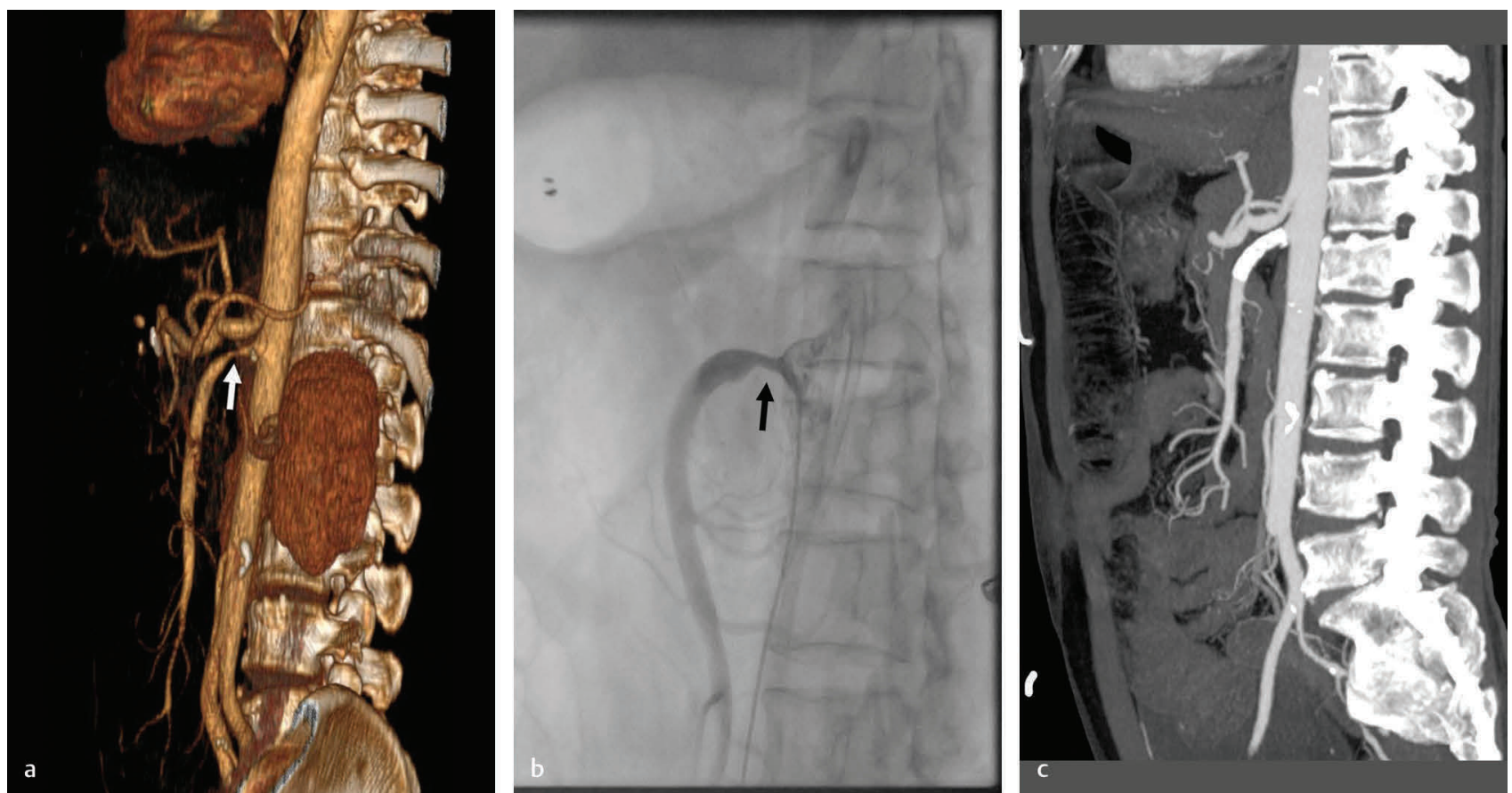

Fig. 1 A 57-year-old man status post SMA stenting presented with massive gastrointestinal bleed. CT angiogram coronal VRT (a) image and catheter angiography (b) lateral image showing significant short segment stenosis of proximal SMA (arrow). Post SMA stenting CT angiogram MIP sagittal image showing restoration of normal luminal caliber (c). CT, computed tomography; MIP, maximum intensity projection; SMA, superior mesenteric artery; VRT, volume rendering technique.

Angiograms were performed with hand injection. A total of $120 \mathrm{~mL}$ of nonionic contrast was used in the procedure. Branches of the gastroduodenal artery (GDA), SMA, and inferior mesenteric artery (IMA) were evaluated sequentially. Digital subtraction was not used because of bowel hyper-peristalsis and respiratory movements. After searching for a bleeder in the GDA, IMA, and most of SMA branches, we found ectasia of the AIPDA. However, no active extravasation was found. The ectatic vessel was super selectively cannulated, an angiogram was performed, and the abnormal ectatic artery was embolized with glue (N-butyl 2 cyanoacrylate) and coils. An initial embolization with $0.5 \mathrm{~mL}$ of $1: 3 \mathrm{mix}-$ ture of Histoacryl glue (B. Braun Melsungen AG) and Lipiodol (Guerbet) was followed by subsequent completion of embolization with four 0.18 " Hilal micro coils $(2 \times 20 \mathrm{~mm}, 2 \times 20 \mathrm{~m}$, $3 \times 50 \mathrm{~mm}$, and $3 \times 30 \mathrm{~mm}$; Cook Medical Inc.). The ectatic artery was found to be completely excluded from circulation after embolization (-Fig. 2). The rest of the mesenteric vessels and branches were normal on angiogram. The patient was closely monitored in the intensive care unit (ICU) after the procedure and was later discharged in a stable condition with a hemoglobin of $7 \mathrm{~g} \%$. Antiplatelet medications were resumed 5 days after the procedure. Retrospective reassessment of the pre- and post-SMA stenting CTA showed normal AIPDA ( - Fig. 3). At a follow-up visit after 18 months, the patient was doing well, and there was no recurrence of bleeding. No indications of hereditary vascular pathology were detected on evaluation during follow-up. This report was prepared after an institutional review board approval and an informed patient consent.
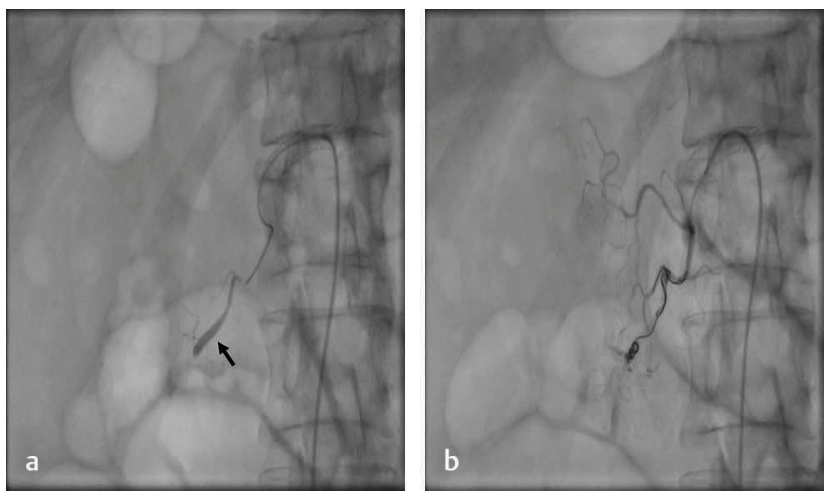

Fig. 2 A 57-year-old man status post SMA stenting presented with massive gastrointestinal bleed. Super selective catheter angiogram of the anterior inferior pancreaticoduodenal artery shows segmental ectasia (black arrow) with few tiny collaterals arising from it (a). Post embolization angiogram of AIPDA showing absent contrast opacification the abnormal vessel and its branches, with preserved flow in the rest of the duodenal and pancreatic vessels (b). AIPDA, anterior inferior pancreaticoduodenal artery; SMA, superior mesenteric artery.

\section{Discussion}

Small intestinal bleeding is an uncommon cause of GI bleeding and accounts for $\sim 5$ to $10 \%$ of all cases. "Overt SB bleeding" refers to patients presenting with either melena or hematochezia with a source of bleeding identified in the small intestine. Recent ACG clinical guidelines proposed the term SB bleeding as replacement for OGIB and reserved obscured GI bleeding for patients not found to have a source of bleeding after performance of standard upper and lower 

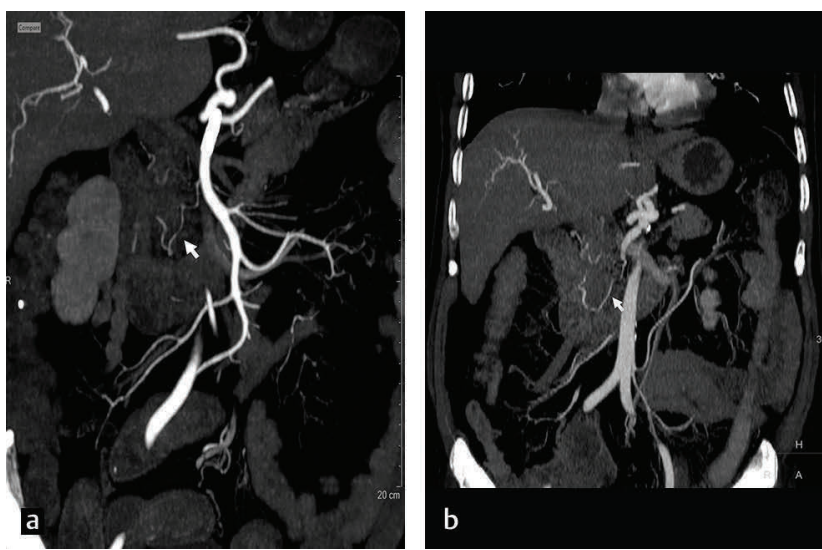

Fig. 3 A 57-year-old man status post SMA stenting who presented with massive gastrointestinal bleed. CTA oblique coronal MIP image (done to detect bleeder) showing normal appearing AIPDA (white arrow) on retrospective analysis (a). Pre stenting CTA coronal MIP image shows normal AIPDA (white arrow) (b). AIPDA, anterior inferior pancreaticoduodenal artery; CTA, computed tomography angiography; MIP, maximum intensity projection; SMA, superior mesenteric artery.

endoscopic examinations, SB evaluation with video capsule endoscopy (VCE) and/or enteroscopy, and radiographic testing. In 85 to $90 \%$ of OGIB cases, source can be identified; however, in 5 to $10 \%$, no source can be found. Sources of bleeding are difficult to diagnose in OGIB, especially if arising from the small intestine. ${ }^{1,2}$ Active overt obscured GI bleeding was seen in present case with melena, fresh clots in distal duodenum, and negative colonoscopy and CTA.

Approximately $75 \%$ cases of OGIB have SB pathologies. Vascular lesions of SB accounts for $~ 70 \%$ of OGIB cases in the Western population and ulcerations account for around $45 \%$ in the Asian population. Other causes include various neoplasms (carcinoid, gastrointestinal stromal tumor [GIST], adenocarcinoma, etc.) and rare hereditary diseases such as hereditary hemorrhagic telangiectasia. ${ }^{3}$

Angiography can detect bleeding at a rate of more than $0.5 \mathrm{~mL} / \mathrm{min}$. Angiographic localization of the source of bleeding varies from less than 50 to $75 \%$, depending on the rate of bleeding during angiography. ${ }^{3}$ CTA can detect bleeding at rates as low as $0.3 \mathrm{~mL} / \mathrm{min}$ and has a sensitivity of 50 to $86 \%$ and specificity of 92 to $95 \%$ for identifying lesions responsible for GIB. ${ }^{4}$ In the present case, the source of bleeding was from a vascular lesion (ectasia of the AIPDA) in pancreaticoduodenal arterial arcade. This vascular lesion was detected on super selective catheter-based angiogram and was successfully embolized. AIPDA was of normal caliber on CTA.

True aneurysms of pancreaticoduodenal arterial arcade are uncommon and account for $2 \%$ of all visceral aneurysms. These true aneurysms are most commonly due to celiac axis stenosis or occlusion. Other etiologies include vasculitis, fibromuscular dysplasia, and congenital collagen disorders. ${ }^{5}$ Hemodynamically significant stenosis of celiac axis causes increased flow through the pancreaticoduodenal arterial arcade and leads to the formation of true aneurysms. Most of these patients are treated with coil embolization when symptomatic. ${ }^{6-8}$ Association of SMA stenosis with pancreaticoduodenal artery and GDA aneurysm is rare. ${ }^{9-13}$ Moore et al reported that the association of pancreaticoduodenal artery aneurysms is more common with celiac axis stenosis than SMA stenosis compared with GDA aneurysms. ${ }^{13}$ One of these case reports have mentioned association of aneurysm of anterior superior pancreaticoduodenal artery with complete occlusion of the superior mesenteric artery. ${ }^{9}$ Most of these cases are treated surgically; however, one case was treated by endovascular method. ${ }^{11}$ In the present case, hemodynamic alteration due to significant SMA stenosis possibly led to the formation of AIPDA ectasia. It was successfully embolized when the patient presented with SB bleeding post SMA stenting possibly due to antiplatelet medication.

Vascular lesions can be treated by various embolic materials. Coils are most commonly used. Because of rich collateral circulation of the upper GI tract, both proximal and distal vascular embolization should be performed to prevent retrograde filling and persistent bleeding from the collaterals. Coils when combined with polyvinyl alcohol particles or Gelfoam are associated with lower recurrent bleeding rates compared with the usage of coils alone. The reported clinical success rate of embolization for upper GIB varies from 44 to $100 \%$. Use of cyanoacrylate glue in GI hemorrhages is promising because of its ability to occlude vessels beyond the most distal site of microcatheter advancement. Glue can also be used in hemodynamically unstable patients as the time required to occlude the vessel is short. Lastly, glue is more efficient in obliteration of pseudoaneurysms with complex morphology. ${ }^{4}$ In our case, cyanoacrylate glue was used to embolize the small collaterals connected to ectatic vessel, and coils were used to fill the ectatic sac and block the inflow from inferior pancreaticoduodenal artery.

\section{Conclusion}

Management of obscure GI bleed is a clinical challenge and requires both endoscopy and imaging evaluation to locate the source. A carefully performed conventional angiogram and embolization can effectively diagnose and treat such rare cases. Gastroduodenal and pancreaticoduodenal arterial arcade ectasias/aneurysms in a patient with celiac axis stenosis/occlusion are a known entity; however, association of these aneurysms with SMA stenosis is rare. SMA stenosis with ectasia/aneurysm of the inferior pancreaticoduodenal artery in specific has not been reported so far.

\section{Conflict of Interest}

None.

\section{References}

1 Bresci G. Occult and obscure gastrointestinal bleeding: causes and diagnostic approach in 2009. World J Gastrointest Endosc 2009;1(1):3-6

2 Gerson LB, Fidler JL, Cave DR, Leighton JA. ACG clinical guideline: diagnosis and management of small bowel bleeding. Am J Gastroenterol 2015;110(9):1265-1287, quiz 1288

3 Pasha SF, Hara AK, Leighton JA. Diagnostic evaluation and management of obscure gastrointestinal bleeding: a changing paradigm. Gastroenterol Hepatol (N Y) 2009;5(12):839-850 
4 Ramaswamy RS, Choi HW, Mouser HC, et al. Role of interventional radiology in the management of acute gastrointestinal bleeding. World J Radiol 2014;6(4):82-92

5 Antoniak R, Grabowska-Derlatka L, Nawrot I, Cieszanowski A, Rowiński O. Aneurysms of peripancreatic arterial arcades coexisting with celiac trunk stenosis or occlusion: single institution experience. BioMed Res Int 2017;2017:1645013

6 Kallamadi R, Demoya MA, Kalva SP. Inferior pancreaticoduodenal artery aneurysms in association with celiac stenosis/ occlusion. Semin Intervent Radiol 2009;26(3):215-223

7 Ikeda O, Tamura Y, Nakasone Y, Kawanaka K, Yamashita Y. Coil embolization of pancreaticoduodenal artery aneurysms associated with celiac artery stenosis: report of three cases. Cardiovasc Intervent Radiol 2007;30(3):504-507

8 Dave B, Sharma A, Kwolek C, Demoya M, Wicky S, Kalva S. Percutaneous transcatheter arterial embolization of inferior pancreatico-duodenal artery aneurysms associated with celiac artery stenosis or occlusion. Catheter Cardiovasc Interv 2010;75(5):663-672
9 Ichinokawa M, Iwai K, Matsumura Y, et al. A case of the anterior superior pancreaticoduodenal artery aneurysm with complete occulusion of the superior mesenteric artery. Jpn J Gastroenterol Surg 2006;39:1678-1682

10 Kimura C, Adachi H, Yamaguchi A, Ino T. A case of an unruptured inferior pancreaticoduodenal artery aneurysm with celiac artery occulusion and superior mesenteric artery stenosis. Jpn J Vasc Surg 2009;18:691-694

11 Ikoma A, Nakai M, Sato M, et al. Inferior pancreaticoduodenal artery aneurysm treated with coil packing and stent placement. World J Radiol 2012;4(8):387-390

12 Gouny P, Fukui S, Aymard A, et al. Aneurysm of the gastroduodenal artery associated with stenosis of the superior mesenteric artery. Ann Vasc Surg 1994;8(3):281-284

13 Moore E, Matthews MR, Minion DJ, et al. Surgical management of peripancreatic arterial aneurysms. J Vasc Surg 2004;40(2):247-253 